\title{
PAT2Math + Handwriting: Evoluindo Sistemas Tutores de Matemática com reconhecimento da escrita à mão
}

\author{
Felipe de Morais ${ }^{1}$, Patrícia A. Jaques ${ }^{1}$ \\ ${ }^{1}$ Programa de Pós Graduação em Computação Aplicada (PPGCA) \\ Universidade do Vale do Rio dos Sinos (UNISINOS), São Leopoldo - RS - Brasil \\ felipedemoraisfm@hotmail.com, pjaques@unisinos.br
}

\begin{abstract}
Intelligent Tutoring Systems (ITSs) for Math still use traditional methods of input, such as computer keyboard and mouse. This strategy exposes students to a high extraneous cognitive load, due to the way the students are used to work, what could impair their learning. This paper presents a tool for Math ITSs that uses the student handwriting as data input. This tool was integrated into ITS PAT2Math, an algebraic ITS, for an experimental evaluation in a public school with 48 students. It followed a design of control and experimental groups with pre and post-tests and a post questionnaire. Although no difference between groups was found, questionnaire responses suggest that handwriting provides a greater facility to the insertion of equations into ITSs for Math when compared with traditional methods (i.e., keyboard and mouse).
\end{abstract}

Resumo. Sistemas Tutores Inteligentes (STIs) de Matemática ainda usam os métodos tradicionais de entrada, como o teclado e mouse do computador. Essa forma de interação expõe os alunos a uma Carga Cognitiva Irrelevante extra, por envolver recursos com os quais os alunos não estão acostumados, o que pode prejudicar a aprendizagem. Este artigo apresenta uma ferramenta para STIs de Matemática que utiliza a escrita à mão dos alunos como entrada de dados. Essa ferramenta foi integrada ao STI algébrico PAT2Math para uma avaliação experimental com 48 alunos de uma escola pública, que seguiu um desenho de grupo de controle e grupo experimental com pré e pós testes e um pós questionário. Embora não tenha sido possivel identificar uma diferença estatística entre os grupos, as respostas dos questionários sugerem que o uso da escrita à mão provê uma maior facilidade para a inserção de equações em STIs de Matemática, quando comparada com teclado e mouse.

\section{Introdução}

Atividades que proporcionam uma maior interatividade ao aluno, possibilitandoo de fazer ao invés de apenas ver, resultam em uma melhora na aprendizagem [Koedinger et al. 2015]. Os Sistemas Tutores Inteligentes (STI) são softwares educacionais que têm a capacidade de proporcionar essa interatividade, pois são capazes de auxiliar o aluno individualmente em suas tarefas. Diferentemente de outros softwares educacionais, os STIs são especialistas em uma determinada área e possuem o conhecimento para fornecer uma tutoria individualizada ao aluno. Eles têm se mostrado quase tão efetivos quanto a tutoria humana individual [VanLehn 2011].

Este artigo descreve o uso de reconhecimento da escrita à mão como método de entrada de dados em STIs de Matemática, mais precisamente nas equações de primeiro 
VI Congresso Brasileiro de Informática na Educação (CBIE 2017)

Anais do XXVIII Simpósio Brasileiro de Informática na Educação (SBIE 2017)

grau. Essas equações são sentenças que contém uma relação de igualdade e utilizam uma notação matemática representada através de símbolos, números e letras. Elas possuem apenas uma variável e tem o objetivo de identificar um valor para esta variável de modo a equilibrar a equação.

Para solucionar equações de primeiro grau, utiliza-se as operações matemáticas básicas (adição, subtração, multiplicação e divisão). Entretanto, devido a restrições de entrada do teclado, nem sempre é simples digitar expressões matemáticas em um ambiente de aprendizagem, geralmente obrigando os alunos a seguirem um conjunto complexo de passos [Anthony et al. 2012]. Por exemplo, em um determinado STI, para inserir uma fração em uma equação, o aluno precisa seguir 13 passos. Este processo de 13 passos pode parecer intuitivo para aqueles que estão usando o sistema por um longo tempo, usuários experientes, mas para estudantes iniciantes no sistema, esta é considerada uma tarefa complexa e que demanda tempo.

Ao expor o aluno a processos complexos e longos em uma tarefa irrelevante ao seu objetivo, que é resolver uma equação, o sistema impõe uma Carga Cognitiva Irrelevante extra [Sweller 2010]. Isso faz com que o aluno ocupe sua Memória de Trabalho com restrições e procedimentos da interface gráfica ao invés de raciocinar sobre a resolução das equações em si [Oviatt et al. 2006].

A Carga Cognitiva está associada com a complexidade do conteúdo, ou informação, a ser estudado e com a complexidade na forma que este conteúdo é apresentado ao aluno. Um determinado conteúdo que está relacionado a vários outros conteúdos é considerado de alta complexidade. Tal conteúdo possui uma alta Carga Cognitiva Intrínseca. A Carga Cognitiva Irrelevante está relacionada com a maneira em que o conteúdo é apresentado ao aluno. Assim, quanto mais o aluno precisa raciocinar sobre a forma como o conteúdo é apresentado, menos ele vai raciocinar sobre o conteúdo a ser aprendido. Em STIs, a Carga Cognitiva Irrelevante pode ser imposta pela Interface Gráfica do sistema. As interfaces que fogem muito da realidade fazem com que os estudantes experienciem uma alta Carga Cognitiva Irrelevante, a qual afeta diretamente a velocidade em que a tarefa é realizada, o foco de atenção, o controle metacognitivo, a exatidão na solução de problemas e a memória [Oviatt et al. 2006].

Esse trabalho tem como objetivo diminuir essa Carga Cognitiva Irrelevante, de modo que o aluno não seja forçado a alterar a maneira que está acostumado a trabalhar com Matemática. Desde as séries inicias, os alunos utilizam lápis e papel para resolver problemas matemáticos. A ideia é utilizar essa estratégia, que se tornou intuitiva aos alunos, como método de entrada das equações em STIs. Para isso, os STIs devem possuir a habilidade de reconhecer a escrita à mão do aluno através de uma imagem.

\section{Trabalhos Relacionados}

O objetivo desta seção é descrever o estado da arte de STIs que utilizam técnicas de reconhecimento da escrita à mão para entrada de dados do sistema. Carnegie Learning's Cognitive Tutor é um STI de álgebra que utiliza do reconhecimento da escrita à mão online para a entrada das equações no sistema. Os autores relatam que a utilização da escrita à mão é mais rápida para a escrita de equações do que a utilização do teclado e mouse. Em um de seus trabalhos, eles relatam que essa diferença foi duas vezes mais rápida [Anthony et al. 2007]. Em seu trabalho mais recente, os autores deram um enfoque maior 
VI Congresso Brasileiro de Informática na Educação (CBIE 2017)

Anais do XXVIII Simpósio Brasileiro de Informática na Educação (SBIE 2017)

para o aumento da precisão no reconhecimento. Para isso, eles adicionaram informações sobre o contexto, obtidas através do Modelo do Aluno [Anthony et al. 2012].

Newton's Pen II (NP2) [Lee et al. 2012] é um STI para física estática, que utiliza o reconhecimento da escrita à mão online para fornecer uma interface mais natural. $\mathrm{Na}$ parte de resolução das equações de Física, o aluno escreve os passos de resolução utilizando uma caneta específica, chamada de stylus pen, e o sistema verifica os passos de resolução inseridos pelo aluno. Foi realizada uma avaliação com mais de 100 estudantes de Engenharia, em que os alunos responderam um survey com questões sobre usabilidade do sistema e sua utilidade para aprendizagem. As perguntas obtiveram respostas acima da média, ilustrando a aceitação e preferência dos alunos pelo sistema proposto.

AnalyticalInk [Kang et al. 2016] é um ambiente que utiliza uma interface interativa com reconhecimento da escrita à mão online, que busca auxiliar os alunos a entenderem conceitos geométricos e resolver exercícios de álgebra e geometria. O sistema fornece problemas em uma forma textual, com algumas palavras chave em destaque. O aluno utiliza essas informações para resolver os problemas, podendo arrastar as informações para a parte de resolução das equações. Ele é capaz de fornecer um feedback visual, referente aos passos de resolução escritos à mão pelos alunos com o uso de um tablet e uma stylus pen. Uma avaliação qualitativa com 10 alunos que utilizaram o sistema evidenciou que o sistema é útil para guiar na resolução de problemas geométricos e algébricos.

Todos os trabalhos relacionados utilizam a escrita à mão como forma de inserção das informações nos sistemas. Porém, o trabalho desenvolvido se diferencia em três principais aspectos: o feedback fornecido a cada passo na resolução dos exercícios, a edição da escrita à mão em tempo real e a não obrigatoriedade de um dispositivo especial.

\section{Uma ferramenta de escrita à mão como entrada de dados para STIs}

Foi desenvolvido um aplicativo web (web app) que pode ser acessado por smartphones, tablets e computadores através de um navegador web. Essa ferramenta contempla a integração de dois trabalhos: o STI PAT2Math e o reconhecedor de escrita à mão online MyScript Math. MyScript fornece um kit de desenvolvimento web, permitindo integrar um elemento gráfico de reconhecimento de escrita à mão com a ferramenta desenvolvida [Mouchere et al. 2014]. Assim, quando o aluno escreve neste elemento gráfico, a ferramenta envia os dados ao servidor do MyScript que extrai informações estáticas e dinâmicas da escrita à mão, insere essas informações em uma Rede Neural Multicamadas e obtém uma lista de probabilidade de símbolos. Após uma análise de relação espacial sobre a escrita e essa lista, o MyScript retorna a escrita reconhecida em formato de texto. Ao receber este texto, a ferramenta exibe a equação para o aluno de maneira multidimensional.

Para a implementação do web app foi utilizado um framework de desenvolvimento de aplicações móveis chamado Apache Cordova. Com este framework é possível desenvolver aplicações para dispositivos móveis utilizando as linguagens de programação para desenvolvimento web, CSS, HTML e JavaScript, ao invés de depender de plataformas específicas de desenvolvimento para cada tipo de dispositivo (iPhone, Android, BlackBerry, etc.). Atualmente, o servidor do STI PAT2Math suporta a comunicação com páginas web, pois utiliza uma interface web, desenvolvida com as linguagens de programação web, CSS, HTML e JavaScript, o que simplificou a a integração com a ferramenta proposta. 
VI Congresso Brasileiro de Informática na Educação (CBIE 2017)

Anais do XXVIII Simpósio Brasileiro de Informática na Educação (SBIE 2017)

\subsection{Funcionamento}

Inicialmente, o aluno acessa a ferramenta proposta através de um dispositivo móvel ou de um computador e faz login no STI PAT2Math com sua conta de usuário (Figura 1.A). Caso ele ainda não tenha uma conta, o cadastro pode ser realizado. Ao efetuar o login, o aluno está enviando suas credenciais para o Módulo Tutor, que por sua vez valida essas credencias com o Módulo do Aluno e, caso válidas, retorna todos os seus dados para o Módulo de Comunicação Móvel. Após o retorno do Módulo Tutor, o aluno terá acesso a uma lista contendo seus planos de aula e tarefas já realizadas e a realizar.

Ao acessar um plano, será exibida uma lista de equações de primeiro grau (Figura 1.B). Caso o aluno já tenha resolvido alguma delas, esta estará salva no Módulo do Aluno e poderá ser visualizada novamente. Caso o aluno ainda não tenha resolvido a equação, esta será exibida na parte superior da tela, como sendo a equação inicial (Figura 1.C1). Na parte inferior da tela, há um quadro em que o aluno poderá utilizar seu dedo para escrever os passos de resolução para a equação inicial (Figura 1.C-8). Enquanto ele vai escrevendo os números e símbolos matemáticos, a ferramenta vai exibindo o que foi reconhecido (Figura 1.C-3). Para isso, a ferramenta fica em constante comunicação com o Módulo de Reconhecimento de escrita à mão, que utiliza o MyScript. Antes de verificar se o passo escrito pelo aluno está correto, a ferramenta já possui a escrita à mão dele reconhecida e convertida em um formato compatível com o STI PAT2Math.

Uma vez finalizada a escrita do passo de resolução, o aluno deve pressionar o botão de verificação (Figura 1.C-4). A ferramenta então envia o passo escrito ao Módulo Tutor que o repassa ao Módulo Especialista para correção, além de salvar as informações no Módulo do Aluno. Um feedback é retornado ao Módulo Tutor que o envia à ferramenta para exibição (Figura 1.C-2). Caso a resposta do aluno esteja correta, a ferramenta permite que ele prossiga para o próximo passo de resolução e assim sucessivamente até identificar o resultado da equação. Caso o passo esteja incorreto, o sistema permite que a escrita seja editada em tempo real. A interface ainda possui botões de auxílio à escrita (Figura 1.C-5,6,7) e de navegação do aplicativo (Figura 1.C-9,10).
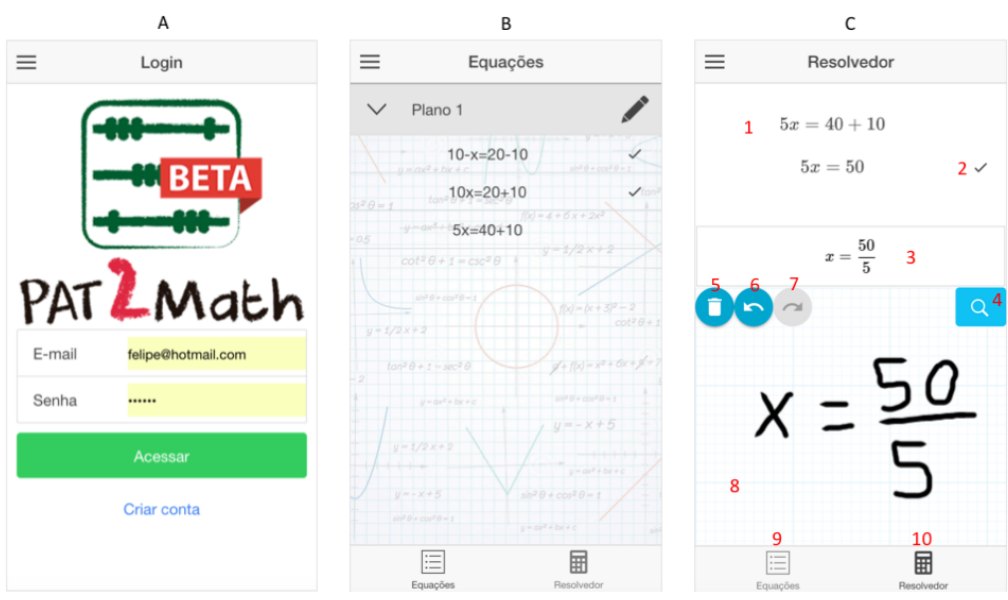

Figura 1. Capturas de tela da ferramenta desenvolvida 
VI Congresso Brasileiro de Informática na Educação (CBIE 2017)

Anais do XXVIII Simpósio Brasileiro de Informática na Educação (SBIE 2017)

\section{Avaliação}

A avaliação teve como objetivo verificar se a escrita à mão, como entrada de dados de um STI de Matemática, leva a uma melhora no desempenho devido à diminuição da carga cognitiva irrelevante, bem como a facilidade de uso da ferramenta, em relação ao método tradicional de entrada de dados de STIs (teclado e mouse). O desempenho de aprendizagem é uma medida indireta e objetiva da carga cognitiva do aluno [Brunken and Plass 2003]. O desenho típico aplicado em experimentos que buscam essa medida é comparar duas diferentes versões de um software educacional de um mesmo conteúdo. Como o conteúdo é o mesmo nos dois sistemas, assume-se que a carga cognitiva intrínseca (relacionada ao conteúdo) é igual, e assim qualquer desempenho diferenciado se deve a uma mudança na carga cognitiva irrelevante [Brunken and Plass 2003].

Dois grupos distintos utilizaram o STI PAT2Math. Os alunos do grupo de controle utilizaram a versão atual do STI PAT2Math, inserindo os passos de resolução das equações através do uso do teclado e mouse do computador. Por outro lado, os alunos do grupo experimental utilizaram a ferramenta desenvolvida, inserindo os passos de resolução das equações com a própria escrita à mão. A Figura 2 ilustra o desenho experimental empregado na avaliação.
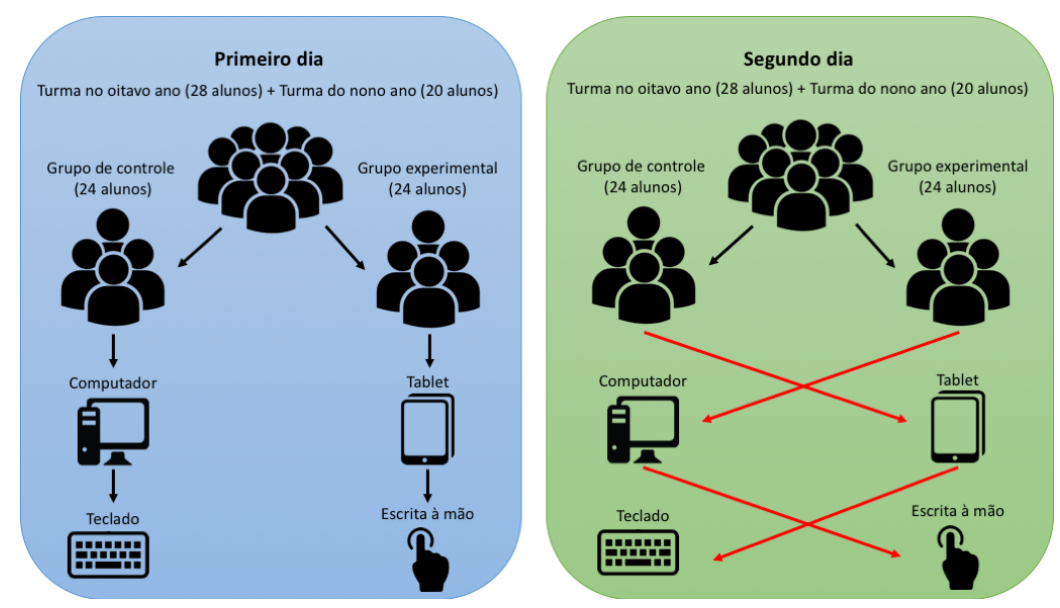

Figura 2. Desenho experimental de avaliação da ferramenta desenvolvida

A ferramenta desenvolvida foi submetida a uma avaliação em duas turmas de uma escola pública na cidade de Parobé (RS). Participaram da avaliação uma turma do oitavo ano do ensino fundamental, com 28 alunos, e uma turma do nono ano do ensino fundamental, com 20 alunos, resultando em um total de 48 alunos com idades entre 13 e 16 anos. Os responsáveis de todos os alunos assinaram um termo de consentimento, permitindo que os dados gerados pelos seus filhos fossem coletados em prol desta pesquisa. Essas turmas foram selecionadas por terem o conteúdo de equações de primeiro grau em seu plano de estudo, conforme definido pelo Ministério da Educação (MEC). Os alunos de cada turma foram distribuídos aleatoriamente em dois grupos, controle e experimental. A avaliação ocorreu em dois dias, sendo que cada turma utilizou o STI PAT2Math durante uma hora e trinta minutos por dia de avaliação. Para a obtenção de dados, foi realizado um teste antes dos alunos utilizarem o sistema, chamado de pré-teste, e um teste após a intervenção, chamado de pós-teste, com ambas as turmas agrupadas. Esses testes seguiam o mesmo nível de dificuldade e tinham como objetivo identificar se os alunos alcançaram 
VI Congresso Brasileiro de Informática na Educação (CBIE 2017)

Anais do XXVIII Simpósio Brasileiro de Informática na Educação (SBIE 2017)

algum ganho na aprendizagem após utilizarem o sistema. Ao final da avaliação, foi aplicado um questionário com o intuito de capturar a opinião dos alunos sobre o sistema.

Para a realização da avaliação, os alunos utilizaram o laboratório de informática da escola, sendo que esse laboratório possuía apenas 14 computadores em funcionamento. Além disso, a conexão com a internet do laboratório era muito lenta. A mesma rede que conectava os computadores era utilizada pela rede sem fio da escola e o único local onde o sinal da rede sem fio alcançava era dentro do laboratório. Sendo assim, ambos os grupos de controle e experimental realizaram a avaliação dentro do mesmo laboratório de informática. Conforme planejado, o grupo de controle deveria utilizar o STI PAT2Math através de um computador e o grupo experimental deveria utilizar a ferramenta desenvolvida através de um dispositivo sensível ao toque. Na turma do nono ano havia 28 alunos, sendo assim, seria necessário 14 computadores para o grupo de controle e 14 dispositivos sensíveis ao toque para o grupo experimental. Foi optado por utilizar tablets como dispositivos sensíveis ao toque por possuírem uma tela maior que um smartphone.

A avaliação ocorreu da seguinte maneira. No primeiro dia de avaliação, ambas as turmas resolveram um pré-teste durante o primeiro período de aula (cada período possui 50 minutos). No segundo e terceiro período, o grupo de controle da turma do oitavo ano, contendo 13 alunos, utilizou o teclado e mouse dos computadores para resolver as equações do STI PAT2Math; um aluno para cada computador. O grupo experimental da mesma turma, com 15 alunos, utilizou os dedos para escrever as equações nos tablets para resolver as equações na ferramenta desenvolvida; um aluno para cada tablet. Os alunos utilizaram seus respectivos sistemas por uma hora e trinta minutos, havendo um tempo de 10 minutos para organização dos alunos e login no sistema. Após o término do terceiro período, os alunos da turma do oitavo ano retornaram às suas salas de aula. No quarto e quinto períodos, ainda do primeiro dia, o mesmo procedimento foi realizado, porém com a turma do nono ano. A única diferença de uma turma para outra foi no número de alunos. No grupo de controle havia 11 alunos e no grupo experimental havia 9 alunos. Após o término do quinto período, os alunos foram liberados pela escola para irem para casa.

No segundo dia de avaliação, foi invertido o dispositivo usado pelos alunos. O grupo de controle da turma do oitavo ano, utilizou o teclado dos tablets para resolver as equações do STI PAT2Math, e não computadores; um aluno para cada tablet. Por outro lado, o grupo experimental da mesma turma, utilizou o mouse do computador para escrever as equações na ferramenta desenvolvida. Um aluno para cada computador. Os alunos do oitavo ano usaram o STI PAT2Math dessa forma durante o primeiro e segundo períodos e após retornaram à sua sala de aula.

Esse desenho experimental foi escolhido pois havia uma ameaça à validade do experimento. Esta ameaça estava relacionada ao fato de que os tablets pudessem causar algum tipo de motivação diferenciada nos alunos. Essa motivação influenciada pelos dispositivos, e não pelo método de entrada das equações, poderia afetar o resultado da avaliação. Essa inversão de dispositivos entre o grupo de controle e o grupo experimental teve o objetivo de equilibrar essa motivação, sendo que assim todos os alunos de ambas as turmas pudessem utilizar ambos os equipamentos. No entanto, cada aluno sempre utilizou o mesmo método de entrada (escrita à mão ou teclado e mouse).

No terceiro e quarto períodos, do segundo dia, o mesmo procedimento de trocar 
VI Congresso Brasileiro de Informática na Educação (CBIE 2017)

Anais do XXVIII Simpósio Brasileiro de Informática na Educação (SBIE 2017)

o dispositivo eletrônico, do primeiro e segundo períodos, foi realizado, porém com a turma do nono ano. Após o termino do quarto período, os alunos da turma do nono ano retornaram às suas salas de aula. Com todos os alunos em suas respectivas salas de aula, foi aplicado o pós-teste. O pré e o pós-teste seguiam o mesmo nível de complexidade das questões. Após o término do pós-teste, todos os alunos responderam um questionário, que tinha como objetivo identificar a opinião dos alunos em relação ao sistema utilizado.

O questionário, assim como os pré e pós-testes, era o mesmo para todos os alunos, independente do aluno ser do grupo de controle ou do grupo experimental. Tal distinção foi realizada somente após o término da avaliação. Sendo assim, não houve influência por parte do professor na correção dos testes. O critério de correção dos testes, adotado pelo professor, foi o mesmo para todos os alunos nos pré e pós-testes. Cada teste contia 12 questões que valiam 1 ponto cada, resultando em um total de 12 pontos. À cada questão correta o aluno ganhava 1 ponto. Caso a questão estivesse errada, mas o professor identificasse que o aluno estava resolvendo da maneira certa e se perdeu por um sinal ou cálculo incorreto, o aluno ganhava 0,5 pontos. Caso a questão estivesse completamente errada ou fosse deixada em branco, o aluno não pontuava nessa questão.

\section{Resultados e discussão}

Conforme descrito na seção de metodologia de avaliação da ferramenta desenvolvida (seção 4), foi realizado um pré-teste antes dos alunos utilizarem o sistema e um pós-teste após. Também foi aplicado um questionário para todos os alunos após a intervenção. Todos os alunos, grupos experimental e controle, resolveram os mesmos testes e responderam o mesmo questionário ao mesmo tempo, em ambas as turmas.

\subsection{Desempenho no Pré e Pós-teste}

Tendo em mente que os testes valiam um total de 12 pontos, foram calculados a média e o desvio padrão para cada um dos grupos no pré e pós-testes. Os dados foram testados para verificar sua normalidade usando Shapiro-Wilk $(p<0.05)$. Após, foi aplicado um teste $t$ para verificar o ganho de aprendizagem e se houve uma diferença de aprendizagem estatisticamente significativa entre os dois grupos.

Um teste $t$ pareado comparou o desempenho entre pré $(M=5,65 ; D P=2,77)$ e o pós-teste $(M=7,62 ; D P=2,98)$ do grupo de controle, com $t(23)=-3$, 311 e $p=$ 0,001 indicando um ganho de aprendizagem significativo. O mesmo teste comparou pré $(M=6,77 ; D P=3,12)$ e pós-testes $(M=7,5 ; D P=2,62)$ do grupo experimental, com $t(23)=-1,5143$ e $p=0,071$ indicando ganho de aprendizagem marginalmente significativo. Foi aplicado a medida de efeito $d$ de Cohen no grupo de controle e no grupo experimental. O grupo de controle obteve $d=0,68$, o que é considerado um efeito médio, com $62 \%$ de sobreposição. O grupo experimental obteve $d=0,25$, o que é considerado um efeito pequeno, com $85 \%$ de sobreposição. Em ambos os grupos, nota-se que houve um aprendizado pelos alunos.

Com o objetivo de identificar se houve um aprendizado mais efetivo no grupo experimental em relação ao grupo de controle, foi utilizado o teste one-way ANCOVA para comparar as notas dos alunos no pós-teste em ambas as condições. Para este teste, as notas do pré-teste foram utilizados como co-variável. Não foi encontrada uma diferença estatisticamente significativa $(F(1,45)=1,173, p=0,284)$ entre as notas do pós-teste 
VI Congresso Brasileiro de Informática na Educação (CBIE 2017)

Anais do XXVIII Simpósio Brasileiro de Informática na Educação (SBIE 2017)

dos grupos de controle e experimental, considerando um nível confiança de 95\%. Ao analisar os dados é possível identificar que a média do pós-teste do grupo experimental é menor que a média do pós-teste do grupo de controle. Porém, a média do pré-teste do grupo experimental é maior que a média do pré-teste do grupo de controle. O teste oneway ANCOVA foi aplicado com o objetivo de reduzir a diferença inicial de conhecimento entre os alunos, usando o pré-teste como co-variável. Como o ANCOVA não encontrou um resultado significativo, é necessário verificar outras possíveis causas para o resultado.

Os autores deste trabalho acreditam que esse resultado deve-se a dois fatores. Primeiro fator, o tempo de utilização do sistema foi curto. Somando o tempo dos dos dias, cada aluno utilizou o sistema por volta de três horas. Segundo fator, os tablets estavam todos conectados na mesma rede sem fio. A internet da escola se apresentava muito lenta, com todos os computadores conectados mais os tablets, a conexão com a internet ficou ainda pior. Essa lentidão afetou principalmente os alunos que utilizavam os dispositivos com conexão sem fio, os tablets. Estes precisavam ter sua escrita reconhecida e precisavam receber um feedback do sistema. Os computadores que acessavam a internet através de cabos tinham uma conexão um pouco mais estável. Sendo assim, o tempo perdido esperando o sistema responder causou uma impaciência aos alunos, fazendo-os dispersar do foco na resolução das equações, afetando diretamente no processo de aprendizagem.

\subsection{Questionário}

Todos os alunos responderam as perguntas de um questionário após a realização do pósteste. As respostas do questionário seguiam uma escala Likert, com resultados variando de 1 a 5 para cada questão. As questões iniciais eram voltadas a informações dos alunos como nome, sexo, idade, gosto por Matemática. As demais questões buscavam identificar o interesse dos alunos em relação à ferramenta desenvolvida.

A questão 6 do questionário perguntava "Você acredita que resolver equações algébricas no STI PAT2Math é:", com respostas variando de Muito interessante (5) a Muito Chato (1). Foi aplicado um teste $t$ independente que verificou a diferença das respostas dos alunos no grupo de controle $(M=4,42, D P=0,70)$ e no grupo experimental $(M=4,87, D P=0,34)$. Com $t(37)=-2,87$ e $p=0,003$, é possível concluir, com segurança estatística, que os alunos consideram a escrita à mão mais interessante que o teclado e mouse para inserção de equações no STI PAT2Math.

A questão 7 questionava se "Ficou mais FÁCIL resolver as equações no STI PAT2Math do que em caderno?", com respostas variando de Sempre (5) a Nunca (1). Foi aplicado um teste $t$ independente que verificou a diferença das respostas dos alunos no grupo de controle $(M=4,08, D P=0,89)$ e no grupo experimental $(M=4,69$, $D P=0,47)$. Com $t(38)=-3,08$ e $p=0,0018$, é possível verificar que os alunos consideram a utilização da escrita à mão mais fácil que a utilização do teclado e mouse.

A questão 8 verificava se "Ficou mais DIVERTIDO resolver as equações no STI PAT2Math do que em caderno?", com respostas variando de Sempre (5) a Nunca (1). Foi aplicado um teste $t$ independente que verificou a diferença das respostas dos alunos do grupo de controle $(M=4,58, D P=0,71)$ e das respostas dos alunos do grupo experimental $(M=4,83, D P=0,49)$. Com $t(44)=-1,45$ e $p=0,076$, não é possível concluir, com segurança estatística, que os alunos consideram a escrita à mão mais divertida do que a utilização de teclado e mouse para inserção das equações no STI 
VI Congresso Brasileiro de Informática na Educação (CBIE 2017)

Anais do XXVIII Simpósio Brasileiro de Informática na Educação (SBIE 2017)

PAT2Math. Porém, é marginalmente provável que tal hipótese seja verdadeira.

A questão 9 questionava "Você acredita que, se pudesse usar o STI PAT2Math em casa para resolver equações, você se motivaria mais para fazer o tema de casa?", com respostas variando de Com certeza sim (5) a Com certeza não (1). Foi aplicado um teste $t$ independente que verificou a diferença das respostas dos alunos no grupo de controle ( $M=4,23, D P=1,14)$ e no grupo experimental. $(M=4,78, D P=0,42)$. Com $t(32)=-2,293$ e $p=0,014$, é possível concluir que os alunos consideram a utilização da escrita à mão mais motivacional do que a utilização do teclado e mouse.

Baseado nas respostas do questionário, pode-se verificar que os alunos do grupo experimental acharam que a utilização da escrita à mão como entrada de dados no STI PAT2Math é mais interessante, mais fácil e provavelmente mais divertida que os métodos tradicionais, teclado e mouse. Além disso, os resultados apontaram que o grupo experimental se motivou mais a usar o STI para fazer seu tema de casa.

\section{Conclusão}

Sistemas Tutores Inteligentes estão se provando cada vez mais eficientes na tarefa de auxiliar os alunos no processo de ensino aprendizagem [VanLehn 2011]. Na área da Matemática, as interfaces gráficas utilizadas para a inserção de dados em STIs utilizam o teclado como método de entrada de dados. Porém, a notação matemática requer mais símbolos do que o teclado do computador oferece. A partir disto, os alunos devem utilizar atalhos e substituições para representar as operações e símbolos matemáticos que desejam inserir. Mas, esse método não é intuitivo. Ele exige que os alunos tenham que raciocinar sobre isso, enchendo sua memória de trabalho com informações irrelevantes, o que faz com que a interface gráfica apresente uma alta carga cognitiva irrelevante, prejudicando a aprendizagem.

A ferramenta desenvolvida tem como objetivo utilizar a escrita à mão do aluno como método de entrada de dados em Sistemas Tutores Inteligentes de Matemática. De modo que o aluno receba um feedback sobre os passos de resolução das equações, a ferramenta desenvolvida utiliza o STI PAT2Math como caso de uso. Dois novos módulos foram adicionados à arquitetura do STI PAT2Math, o Módulo de comunicação móvel e o Módulo de reconhecimento da escrita à mão. O Módulo de reconhecimento da escrita à mão utiliza uma ferramenta de reconhecimento da escrita à mão online já evidenciada e validada na literatura, chamada de MyScript Math. O Módulo de comunicação móvel foi implementado como um web app, sendo assim, o aluno pode acessar a ferramenta através do navegador do seu computador, de um smartphone ou de um tablet.

A ferramenta desenvolvida foi submetida a uma avaliação com duas turmas de uma escola pública. Os alunos de cada turma foram distribuídos aleatoriamente em um grupo de controle e um grupo experimental. O grupo de controle utilizou o STI PAT2Math com sua interface atual, utilizando teclado e mouse no computador e o teclado no tablet, e o grupo experimental utilizou a ferramenta desenvolvida, utilizando a escrita à mão, com os dedos nos tablets e com o mouse nos computadores. Os alunos realizaram um pré-teste antes de utilizar o sistema e um pós-teste após utilizar o sistema. O objetivo da avaliação era verificar se a ferramenta desenvolvida apresentava uma melhora no processo de inserção de dados no STI PAT2Math, proporcionando assim uma carga cognitiva irrelevante menor, resultando em uma melhora na aprendizagem. O desem- 
VI Congresso Brasileiro de Informática na Educação (CBIE 2017)

Anais do XXVIII Simpósio Brasileiro de Informática na Educação (SBIE 2017)

penho de aprendizagem é uma medida indireta e objetiva da carga cognitiva do aluno [Brunken and Plass 2003].

Baseado na comparação dos resultados dos testes, não foi possível encontrar uma significância estatística que houve uma melhora de desempenho do grupo experimental em relação ao grupo de controle. Os autores acreditam que esse resultado se deve à curta duração do experimento, assim como à baixa velocidade na conexão com a internet da escola.

No entanto, as respostas do questionário evidenciam que os estudantes que usaram a escrita à mão acharam mais interessante, mais fácil e provavelmente mais divertido resolver equações no STI PAT2Math. Também foi identificado que os alunos que utilizaram a ferramenta proposta se motivariam mais a estudar em casa utilizando o STI PAT2Math. A escrita à mão parece proporcionar uma facilidade maior para os alunos entrarem com as equações no STI. Desse modo, o aluno precisa raciocinar menos sobre como inserir as equações no sistema e investir seu raciocínio lógico na resolução das equações. Como trabalhos futuros, pretende-se realizar uma avaliação em maior escala, em número de participantes e no período de tempo de utilização da ferramenta, e com uma infraestrutura mais adequada.

\section{Referências}

Anthony, L., Yang, J., and Koedinger, K. R. (2007). Benefits of handwritten input for students learning algebra equation solving. Frontiers in Artif. Intel. and App., 158:521.

Anthony, L., Yang, J., and Koedinger, K. R. (2012). A paradigm for handwriting-based intelligent tutors. Int. J. of Human-Computer Studies, 70(11):866-887.

Brunken, R. and Plass, J. L. (2003). Direct measurement of cognitive load in multimedia learning. Educational Psychologist, 38(1):53-61.

Kang, B., Kulshreshth, A., and LaViola Jr, J. J. (2016). Analyticalink: An interactive learning environment for math word problem solving. In Int. Conf. on Intelligent User Interfaces, pages 419-430. ACM.

Koedinger, K. R., Kim, J., Jia, J. Z., McLaughlin, E. A., and Bier, N. L. (2015). Learning is not a spectator sport. In ACM Conf. on Learning Scale, pages 111-120. ACM.

Lee, C., Jordan, J., Stahovich, T. F., and Herold, J. (2012). Newtons pen ii: an intelligent, sketch-based tutoring system and its sketch processing techniques. In Int. S. on SketchBased Interfaces and Modeling, pages 57-65. Eurographics Association.

Mouchere, H., Viard-Gaudin, C., Zanibbi, R., and Garain, U. (2014). Icfhr 2014 competition on recognition of on-line handwritten mathematical expressions (crohme 2014). In Int. Conf. on Frontiers in Handwriting Recognition, pages 791-796. IEEE.

Oviatt, S., Arthur, A., and Cohen, J. (2006). Quiet interfaces that help students think. In ACM S. on User interface software and technology, pages 191-200. ACM.

Sweller, J. (2010). Element interactivity and intrinsic, extraneous, and germane cognitive load. Educational psychology review, 22(2):123-138.

VanLehn, K. (2011). The relative effectiveness of human tutoring, intelligent tutoring systems, and other tutoring systems. Educational Psychologist, 46(4):197-221. 